ARTICLE

Received 1 Feb 2016 | Accepted 28 Jun 2016 | Published 4 Aug 2016

DOI: $10.1038 /$ ncomms12380

OPEN

\title{
Enhanced hydrogenation activity and diastereomeric interactions of methyl pyruvate co-adsorbed with R-1-(1-naphthyl)ethylamine on $\mathrm{Pd}(111)$
}

Mausumi Mahapatra ${ }^{1}$, Luke Burkholder ${ }^{1}$, Michael Garvey ${ }^{1,2}$, Yun Bai ${ }^{1}$, Dilano K. Saldin ${ }^{3} \&$ Wilfred T. Tysoe

Unmodified racemic sites on heterogeneous chiral catalysts reduce their overall enantioselectivity, but this effect is mitigated in the Orito reaction (methyl pyruvate (MP) hydrogenation to methyl lactate) by an increased hydrogenation reactivity. Here, this effect is explored on a $R$-1-(1-naphthyl)ethylamine (NEA)-modified $\mathrm{Pd}(111)$ model catalyst where temperature-programmed desorption experiments reveal that NEA accelerates the rates of both MP hydrogenation and H/D exchange. NEA + MP docking complexes are imaged using scanning tunnelling microscopy supplemented by density functional theory calculations to allow the most stable docking complexes to be identified. The results show that diastereomeric interactions between NEA and MP occur predominantly by binding of the $\mathrm{C}=\mathrm{C}$ of the enol tautomer of MP to the surface, while simultaneously optimizing $\mathrm{C}=\mathrm{O} \cdots \mathrm{H}_{2} \mathrm{~N}$ hydrogen-bonding interactions. The combination of chiral-NEA driven diastereomeric docking with a tautomeric preference enhances the hydrogenation activity since $\mathrm{C}=\mathrm{C}$ bonds hydrogenate more easily than $\mathrm{C}=\mathrm{O}$ bonds thus providing a rationale for the catalytic observations.

\footnotetext{
${ }^{1}$ Department of Chemistry and Biochemistry, University of Wisconsin-Milwaukee, Milwaukee, Wisconsin 53211, USA. ${ }^{2}$ Applied Research Institute, University of Illinois at Urbana-Champaign, Champaign, Illinois 61820, USA. ${ }^{3}$ Department of Physics, University of Wisconsin-Milwaukee, Milwaukee, Wisconsin 53211, USA. Correspondence and requests for materials should be addressed to W.T.T. (email: wtt@uwm.edu).
} 
$\mathrm{P}$ harmaceuticals and agrochemicals must be produced in an enantiopure form ${ }^{1}$, and are most often synthesized using organometallic catalysts with a chiral stereo-directing ligand at the reaction centre ${ }^{2-5}$; there are currently many examples of highly enantioselective, homogeneous-phase chiral catalysts ${ }^{6}$. However, this approach requires the stringent removal of the heavy metals from the product mixture so that the use of heterogeneous-phase chiral catalysts would therefore be beneficial. Organometallic catalysts can be heterogenized by anchoring them to a support, but such tethered catalysts can suffer from problems such as leaching or reduced enantioselectivity $^{7-9}$. An alternative approach is to implement a strategy analogous to that exploited in homogeneous phase, by directly modifying the catalyst surface using a chiral ligand. Here, the chiral modifiers can be classified as those that act as templates or one-to-one modifiers ${ }^{10}$. Templates function by several chiral adsorbates acting in concert to provide enantioselective reaction sites, while one-to-one modifiers operate via a direct diastereomeric interaction between the modifier and prochiral reactant ${ }^{11}$. However, while all the reaction centres of an organometallic compound are influenced by the chiral ligand, it is a major challenge to modify all reaction sites on an extended surface. As unmodified sites will form racemic products, this reduces the overall enantioselectivity. This could conceivably be addressed by forming an extended template array which exposes only enantioselective pockets, but this has not, as yet, been achieved. Nonetheless, heterogeneous chiral catalysts have been synthesized, in particular for the enantioselective hydrogenation of $\alpha$-ketoesters. For example, the $\mathrm{CH}_{3}-\mathrm{C}=\mathrm{O}$ bond in methyl pyruvate (MP) can be enantioselectively hydrogenated to methyl lactate over cinchona-modified catalysts in the so-called Orito reaction where cinchona operates as a one-to-one modifier ${ }^{12-24}$. In this case, it has been found that hydrogenation rates are substantially increased at chirally modified sites compared with the unmodified catalyst ${ }^{17,22,24-27}$, thereby enhancing the effect of the diastereomeric interaction to produce enantiomeric excesses (ee) approaching $100 \%$. This effect provides a potential solution to this central problem in designing heterogeneous chiral catalysts, but the origin of this rate enhancement is not well understood.

To explore the diastereomeric interactions between MP and chiral modifiers, and to investigate the origins of the enhanced hydrogenation activity, experiments are performed on a model $\operatorname{Pd}(111)$ single-crystal catalyst modified by $R-1$-(1-naphthyl)ethylamine (NEA) since the cinchona alkaloids are difficult to introduce into vacuum. NEA shares the key functional aspects of cinchonidine and has been used as a model chiral modifier ${ }^{28-32}$, where both endo and exo adsorbate conformers have been identified ${ }^{31}$. NEA has also been found to be randomly distributed on surfaces thus implying that NEA should act as a one-to-one modifier to provide a chiral reaction site ${ }^{30,31}$. The chemistry of the simplest $\alpha$-ketoester, MP has been explored on several transition-metal surfaces ${ }^{33-38}$ where it exists as both the enol and keto tautomer (depicted in Supplementary Fig. 4$)^{36,38}$ and the energetics on $\operatorname{Pd}(111)$ have been discussed in detail ${ }^{37}$. However, MP tends to agglomerate on platinum, the most commonly used catalyst for the enantioselective hydrogenation of MP (ref. 39), making it difficult to identify docking complexes between MP and NEA using scanning tunnelling microscopy (STM) on this surface. MP interacts to a lesser extent on $\mathrm{Pd}(111)$, thereby optimizing the possibility of observing interactions between MP and NEA.

The results show that complexes predominantly form with the enol tautomer of MP. The most stable diastereomeric interactions between MP and NEA occur when the $\mathrm{C}=\mathrm{C}$ bond of the enol tautomer binds to the most stable Pd bridge site, while simultaneously optimizing the $\mathrm{C}=\mathrm{O} \cdots \mathrm{H}_{2} \mathrm{~N}$ hydrogen-bonding interactions between NEA and MP. Since $\mathrm{C}=\mathrm{C}$ double bonds hydrogenate significantly more easily than $\mathrm{C}=\mathrm{O}$ bonds ${ }^{40}$, this provides a plausible explanation for the enhanced hydrogenation activity found on chirally modified catalysts.

\section{Results}

Temperature-programmed desorption experiments. To understand the enhancement in hydrogenation activity, we first explore the hydrogenation of MP on NEA-modified $\mathrm{Pd}(111)$ using temperature-programmed desorption (TPD) to establish that a $\operatorname{Pd}(111)$ single crystal adequately reproduces this enhancement effect. A Pd(111) surface was dosed with various coverages of NEA, where the coverages were determined by carbon monoxide site blocking as described previously ${ }^{41}$. The surface was then saturated with atomic hydrogen (31 (1 L (Langmuir) $=$ $1 \times 10^{-6}$ Torr $\cdot s$ ) exposure), and finally saturated with MP. The resulting $45 \mathrm{AMU}$ (the most intense signal of methyl lactate, assigned to the $\mathrm{CH}_{3}-\mathrm{CH}-\mathrm{OH}^{+}$mass spectrometer ionizer fragment) TPD profiles are displayed in Fig. 1, as a function of NEA coverage. The most intense mass spectrometer fragment of $\mathrm{MP}$ is at $43 \mathrm{AMU}$ (from the $\mathrm{CH}_{3}-\mathrm{C}=\mathrm{O}^{+}$fragment), with a small ( $\sim 2 \%$ relative intensity) signal at $45 \mathrm{AmU}$ mass, giving rise to the multilayer MP feature at $\sim 170 \mathrm{~K}$ (ref. 37), which is evident primarily at an NEA coverage of 0.78 monolayers. A clear methyl lactate desorption peak is evident depending on the $R$-NEA coverage, which is centred at $\sim 246 \mathrm{~K}$ for a NEA coverage of 0.49 monolayers, decreasing in temperature to $\sim 225 \mathrm{~K}$ at higher NEA coverages. The assignment of this feature to methyl lactate was confirmed by measuring the signals at other masses. The methyl lactate yield is below the detection limit for NEA coverages of 0.38 monolayers and lower (data not shown), but the methyl lactate yield increases with increasing NEA coverage

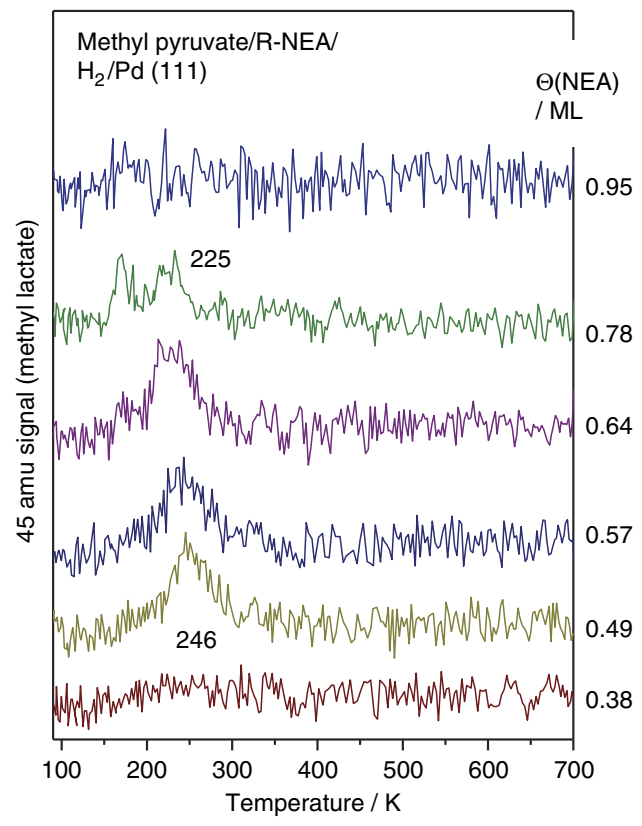

Figure 1 | TPD profiles showing the effect of NEA co-adsorption on MP hydrogenation on Pd(111). The traces show the $45 \mathrm{AMU}$ (methyl lactate) TPD profiles collected following the adsorption of $3 \mathrm{~L}(1 \mathrm{~L}$ (Langmuir) $=$ $\left.1 \times 10^{-6} \mathrm{Torr} \cdot \mathrm{s}\right)$ of MP on a Pd(111) surface precovered with NEA and saturated with hydrogen, all dosed at a sample temperature of $\sim 90 \mathrm{~K}$, as a function of NEA coverage. The NEA coverages are measured by CO site blocking and the values are displayed adjacent to the corresponding desorption profile. 
demonstrating that MP hydrogenation is enhanced by the presence of NEA. The hydrogenation yield decreases to zero once again at the highest NEA coverage as MP adsorption is blocked. The proximity of the methyl lactate desorption temperature (from $\sim 225$ to $246 \mathrm{~K}$ ) to that for ethylene hydrogenation on $\operatorname{Pd}(111)(\sim 250 \mathrm{~K}$, Supplementary Fig. 1, ref. 42) is consistent with it forming by hydrogenation of a $\mathrm{C}=\mathrm{C}$ double bond, indicating that the methyl lactate forms by hydrogenation of the enol tautomer of MP.

To further explore the influence of keto-enol tautomerization and, since H/D exchange occurs for ethylene on group VIII transition metals ${ }^{43}$ including $\operatorname{Pd}(111)$ (Supplementary Fig. 2, Supplementary Table 1), TPD experiments were carried out for MP adsorbed on NEA-precovered $\operatorname{Pd}(111)$ that had been saturated with deuterium. The resulting $46 \mathrm{AMU}$ desorption profiles are shown in Fig. 2 for various NEA coverages. The peak desorption temperature and shape indicate that the feature is due to MP desorption so that the peak is assigned to $d_{3}-M P$ (from the $\mathrm{CD}_{3}-\mathrm{C}=\mathrm{O}^{+}$fragment). The results indicate that $\mathrm{MP}$ has undergone substantial $\mathrm{H} / \mathrm{D}$ exchange, again providing clear evidence of the participation of the enol form of MP in the reaction. No $46 \mathrm{AMU}$ signal is detected in the absence of deuterium (bottom profile, Fig. 2), but H/D exchange occurs even without NEA $(\Theta(\mathrm{NEA})=0$ monolayers $)$. The inset to Fig. 2 shows the integrated area of the $46 \mathrm{AMU}$ signals ratioed to the relative MP coverage, which decreases due to site blocking by NEA, and was measured from the integrated intensity of 43 AMU TPD profiles collected in the same experiment. This ratio increases over the same NEA coverage range as that at which MP hydrogenation is observed (Fig. 1).

NEA Adsorption Site on Pd(111). Previous density functional theory (DFT) calculations have indicated that the naphthyl ring of NEA is located on the so-called dibridge[7] site (Supplementary Fig. 3) 32,44 , while calculations on $\mathrm{Pt}(111)$ have suggested that it adsorbs on the dibridge[6] site (Supplementary Fig. 3$)^{39,45}$. The dibridge[7] site on $\mathrm{Pd}(111)$ was previously

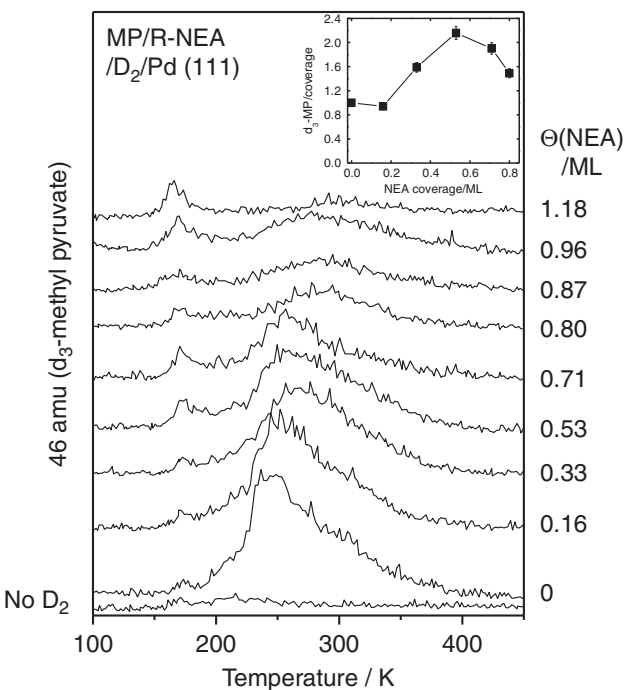

Figure 2 | TPD profiles showing the effect of NEA co-adsorption on hydrogen/deuterium exchange on Pd(111). The traces show the $46 \mathrm{AMU}$ $\left(d_{3}-M P\right)$ TPD profiles collected following the adsorption of $3 \mathrm{~L}$ of MP on a $\mathrm{Pd}(111)$ surface precovered with NEA and saturated with hydrogen, all dosed at a sample temperature of $\sim 90 \mathrm{~K}$, as a function of NEA coverage. The NEA coverages are measured by $\mathrm{CO}$ site blocking and the values are displayed adjacent to the corresponding desorption profile. identified by first calculating the most stable naphthalene adsorption site (dibridge[7] ${ }^{44}$ ) and then by adding an ethylamine group to calculate the final structure. Since the separation between NEA and MP in the docking complex is likely to significantly influence the nature of the diastereomeric interactions, we recalculated the most stable structure of NEA on $\mathrm{Pd}(111)$ adsorbed at various sites and found that the dibridge[6] site was, in fact, also more stable on $\operatorname{Pd}(111)$ than adsorption on the dibridge[7] site, where the exo conformer is more stable by $20 \mathrm{~kJ} \mathrm{~mol}^{-1}$ and the endo structure by $11 \mathrm{~kJ} \mathrm{~mol}^{-1}$ (Supplementary Discussion). To unequivocally establish the correct NEA adsorption site, we measured the low-energy electron diffraction (LEED) intensity versus beam voltage (I/V) curves of NEA on $\mathrm{Pd}(111)$ (Supplementary Fig.18) ${ }^{46}$. NEA on the dibridge[6] site was found to have the lowest Pendry R-factor (0.15 for the dibridge[6] site compared with 0.23 for the dibridge[7] site; Supplementary Table 13). This reveals that the binding of NEA to the surface is not only controlled by the preferred naphthyl adsorption site but is also strongly influenced by the binding of the amine lone pair to a palladium atop site, which then shifts the ring from a dibridge[7] to a dibridge[6] site.

Scanning tunnelling microscopy of docking complexes. The docking complexes formed between prochiral MP and the chiral modifier, NEA are explored by imaging them using STM. Firstprinciples DFT calculations and STM image simulations are used to identify the nature of the docking complexes and to compare the theoretically predicted and experimental distributions of the various types of docking complex, similar to previous approaches to understand chirality transfer on platinum substrates $35,39,47-49$. STM images of co-adsorbed NEA and MP on $\mathrm{Pd}(111)$ were collected at a sample temperature of $\sim 120 \mathrm{~K}$ by first exposing the surface to NEA, followed by dosing with MP. Docking complexes were identified on the surface and the imaging temperature was sufficiently low that the complexes were stable and no dynamic behaviour was observed in consecutive images. Typical highresolution images of the most commonly observed types of complex in which NEA is coordinated to a single MP molecule, are shown in Fig. 3a-c. The complexes were classified by measuring the angle subtended between the region of the NEA molecule due to the naphthyl ring (indicated by yellow lines) and the long axis of the MP molecule adjacent to it (indicated by red lines; Supplementary Fig. 19). In rare cases, two MP molecules were found to coordinate to NEA. An example of this structure is shown in the wider-scan-area image in Fig. 3(d), which also displays well-separated, isolated NEA adsorbed as exo (highlighted in red) and endo (highlighted in blue) conformers. Statistical analyses were performed for multiple images for over 100 clearly identifiable complexes (see Supplementary Discussion for the measurement protocol and sampling error calculations). Other complexes could have been identified in more crowded regions of the surface, but were not included in the statistical analysis because of the difficulty in unequivocally distinguishing a MP molecule from an adjacent naphthyl ring. In all cases, MP was found to adsorb adjacent to the ethylamine functionality.

A histogram showing the experimental distribution of the abundance of the docking complexes is displayed as a function of the measured angles in Fig. 4a. The dihedral angles fall into three well-defined bins designated A $\left(10 \pm 5^{\circ}\right), \quad$ B $\left(45 \pm 5^{\circ}\right)$ and C $\left(70 \pm 5^{\circ}\right)$. The difference between the angles that define each bin is sufficiently large that the images of clearly defined complexes could be unequivocally assigned to each bin. The measured populations are summarized in Table 1 with the associated sampling errors. Approximately 3\% of the docking 

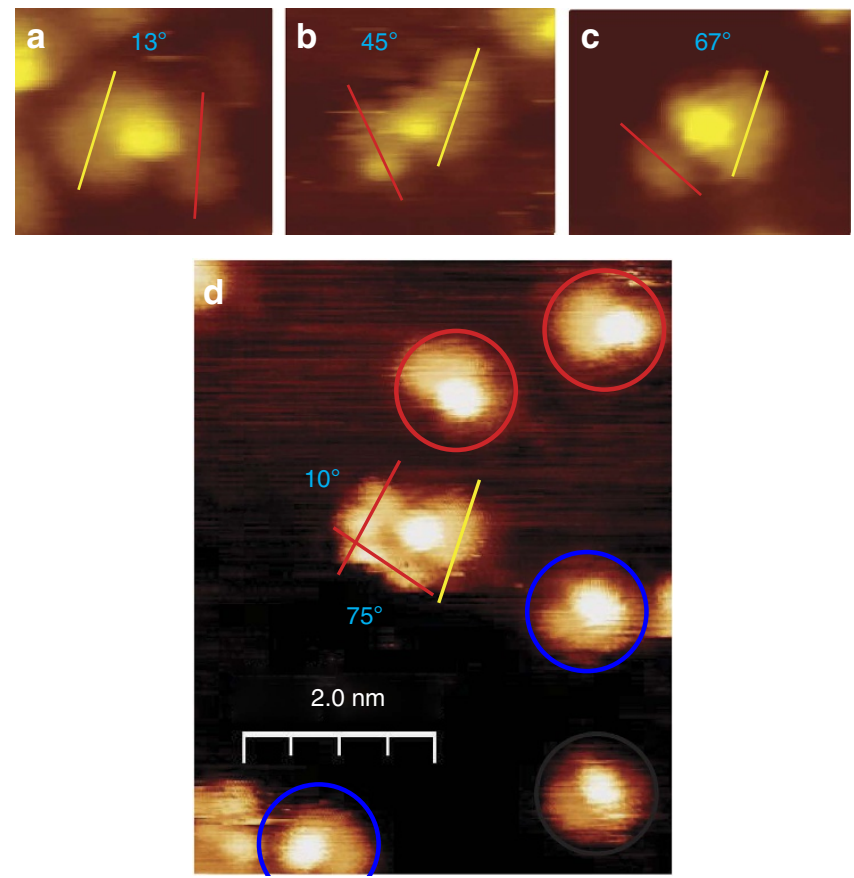

Figure 3 | Example images of docking complexes formed between MP and NEA on Pd(111). The figure shows high-resolution STM images of the docking complexes identified from co-adsorbed NEA and MP on Pd(111) at $120 \mathrm{~K}$. In most cases, a single MP molecule coordinates to NEA. Examples of such docking complexes are shown in $\mathbf{a}-\mathbf{c}$ and are characterized based on the angles between the long axes of the naphthyl group (indicated as yellow lines) and the MP molecule (indicated as red lines). The examples shown, which appear most often, have dihedral angles of $\sim 13^{\circ}$ (a), $45^{\circ}$ (b) and $67^{\circ}$ (c). In rare cases, shown in $\mathbf{d}$, which displays a wider scan area, two MP molecules can coordinate to a NEA molecule. This figure also illustrates that the docking complexes are well separated from isolated NEA molecules, which are highlighted in red (exo conformer) and blue (endo conformer).

complexes were found to include two MP molecules oriented at $10 \pm 5$ and $70 \pm 5^{\circ}$ to the NEA naphthyl ring (Fig. 3d). It was not possible to unequivocally establish the NEA isomer contained in each of the docking complexes due to the proximity of the MP bonded to it.

\section{Discussion}

Co-adsorption of MP with NEA enhances the hydrogenation rate of MP to methyl lactate (Fig. 1), thereby reproducing the effect found for catalytic systems. Adsorbed MP also undergoes significant $\mathrm{H}$-D exchange to form $\mathrm{d}_{3}$-MP (Fig. 2) both on clean $\operatorname{Pd}(111)$ and the NEA-covered surface. Such H-D exchange indicates reaction with the enol tautomer on the surface. H-D exchange also occurs on $\mathrm{Pd}(111)$ in the absence of co-adsorbed NEA, but the proportion of MP undergoing complete exchange increases on NEA-modified Pd(111) (Fig. 2, Inset).

While the STM images identify docking complexes and provide structural information, the resolution is insufficient to distinguish the forms of NEA and MP. Accordingly, the docking of enol and keto tautomers of MP with both the endo and exo isomers of NEA was investigated using DFT calculations by constructing isolated 1:1 complexes of all four combinations with MP adsorption constrained to be proximate to the chiral ethylamine centre on NEA. MP becomes chiral due to removal of the mirror plane by adsorption and can therefore adopt Pro- $R$ or Pro- $S$
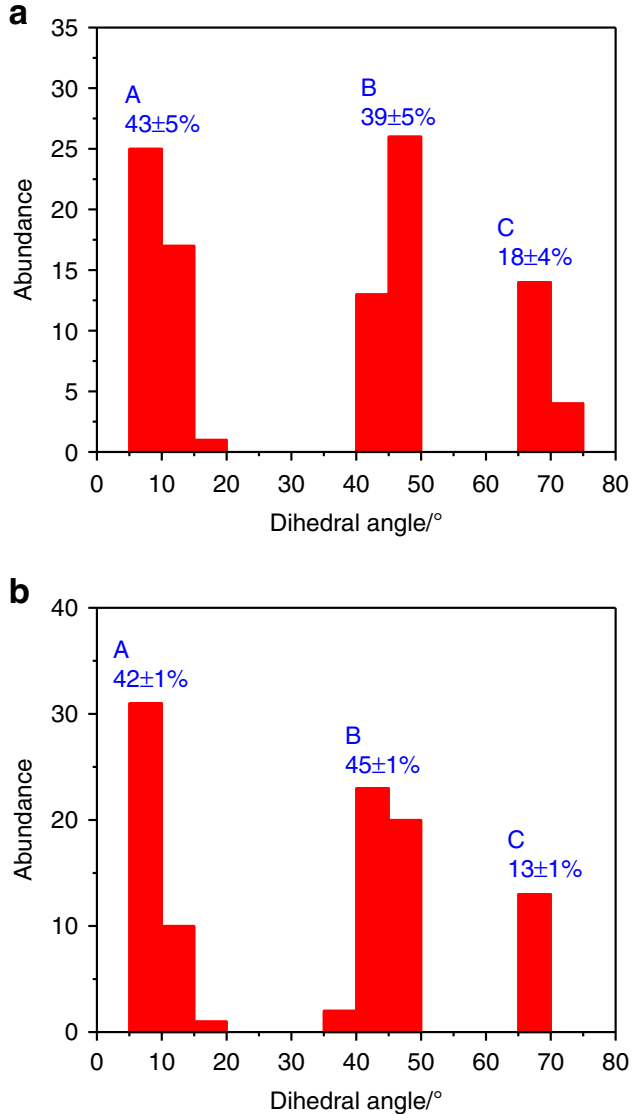

Figure 4 | Comparison of experimental and calculated distributions of docking complexes formed between MP and NEA on Pd(111). The figure displays histograms showing the proportions of (a) experimentally measured and (b) calculated abundances versus the dihedral angles between the naphthyl ring of NEA and the long axis of MP. The results reveal that the complexes fall into three, well-defined bins defined by a range of dihedral angles; $\mathrm{A} ; 10 \pm 5^{\circ}, \mathrm{B} ; 45 \pm 5^{\circ}$ and $\mathrm{C} ; 70 \pm 5^{\circ}$.

configurations. Calculations were therefore carried out for the interaction of both MP enantiomers with NEA. Initial calculations were performed without including van der Waals' interactions. However, since van der Waals forces have been found to provide a large contribution to the binding energies on coinage metal surfaces ${ }^{50}$, the energies and structures of the most stable docking complexes were then calculated by including van der Waals interactions using the method of Tkatchenko and Scheffler ${ }^{51}$, which leads to more stable structures and larger interaction energies in all cases. While DFT calculations of binding energies can have significant systematic errors, it is expected that the relative energies of the same systems in different configurations will be reasonably accurate. Interaction energies were calculated relative to the exo and endo conformers of NEA and the keto tautomer of MP alone on a $6 \times 6 \mathrm{Pd}(111)$ slab (Supplementary Discussion). These results provide an indication of the relative interaction strengths of various docking complexes. Interaction energies of the most stable docking complexes were then calculated by including van der Waals interactions (Supplementary Table 12). The resulting energies were then used to calculate relative equilibrium proportions of each docking complex at a sample temperature of $120 \mathrm{~K}$. Since STM images were collected from multiple experiments over a period of time, the effect of temperature fluctuations of $\pm 10 \mathrm{~K}$ on the equilibrium distributions was also estimated. 
Table 1 | Comparison between measured and calculated proportions of docking complexes.

\begin{tabular}{cccc} 
Dihedral angle range ${ }^{\circ}$ & Measured proportion/\% & Calculated proportion, exo NEA only/\% & Calculated proportion, exo and endo NEA/\% \\
\hline $10 \pm 5$ & $43 \pm 5$ & 28 & $42 \pm 1$ \\
$45 \pm 5$ & $39 \pm 5$ & 48 & $45 \pm 1$ \\
$70 \pm 5$ & $18 \pm 4$ & 24 & $13 \pm 1$
\end{tabular}

Measured proportions of NEA-MP docking complexes that have dihedral angles in the STM images between the naphthyl group of NEA and the long axis of the MP of $10 \pm 5,45 \pm 5$ and $70 \pm 5^{\circ}$, compared with the results of the image analysis of the structures of the docking complexes calculated using DFT for only the exo form of NEA, and then including both the endo and exo forms. The measured proportions include the sampling error and the calculated proportions take into account possible variations in the sample temperature.

Finally, the STM images of the most stable structures were simulated using the Tersoff-Hamman approach $^{52}$ (Supplementary Figs 16 and 17). In all cases, the shapes of the simulated images agree with the experimental images. The dihedral angles between MP and the naphthyl ring of NEA in the simulated images were measured in the same way as for the experiment and fell into the same ranges. The equilibrium distribution of angles in the simulated images of the calculated structures are displayed in Fig. $4 \mathrm{~b}$ and summarized in Table 1, where the errors include the uncertainly in temperature discussed above. Including docking complexes arising from just the exo conformer of NEA yields distributions that are not in good agreement with experiment, while adding complexes with both endo and exo NEA agrees very well. This indicates that the correct structures are reproduced by the DFT calculations and thus provides atomic-scale information on diastereomeric interactions between MP and NEA.

The most stable docking complexes (those with greater than $\sim 8 \%$ abundance) are displayed for exo NEA in Fig. 5 and for endo NEA in Fig. 6, while the remainder of the docking complexes that appear less frequently, but which were nevertheless included in the calculation of the populations, are shown in Supplementary Figs 16 and 17, along with their interaction energies and calculated equilibrium proportions. Figures 5 and 6 also show the simulated STM images and indicate both the calculated interaction energies and equilibrium proportions.

The majority of the docking complexes are with the enol tautomer of MP (78\%), with the remainder interacting with the keto form. Thus, the enhanced hydrogenation activity of NEA-covered Pd(111) compared with the clean surface (Fig. 1) is ascribed to the preferential docking of the enol form of MP where the $\mathrm{C}=\mathrm{C}$ double bond undergoes more facile hydrogenation than the $\mathrm{C}=\mathrm{O}$ bond $^{40}$. The participation of the enol tautomer has been proposed previously for MP hydrogenation on cinchonidine- and cinchonine-modified, supported palladium catalysts $^{53}$. This conclusion is in accord with the similarity in peak temperature for MP hydrogenation on NEA-covered $\mathrm{Pd}(111)$ and for ethylene hydrogenation on clean $\mathrm{Pd}(111)$ (Supplementary Fig. 1). As noted above, MP can be classified into whether reacts to form $R$ - or $S$-methyl lactate and, assuming only the enol form hydrogenates, predicts an ee of $R$-methyl lactate in the presence of $R$-NEA of $\sim 92 \%$. The calculated proportion of the docking complexes that form with the exo isomer of NEA is $\sim 54 \%$, with the remainder docking with the endo form. This ratio is close to that found for NEA adsorbed alone on $\operatorname{Pd}(111)$, where the $57 \pm 3 \%$ adsorbs as the exo conformer $^{31}$

All the most stable complexes (Figs 5 and 6) bind to the enol MP tautomer in a cis configuration ${ }^{38}$, with the $\mathrm{C}=\mathrm{C}$ double bond located at Pd-Pd bridge sites. The methylene hydrogens are distorted from a planar geometry as found for ethylene on $\mathrm{Pd}(111)^{54}$. Docking is dominated by $\mathrm{C}=\mathrm{O} \cdots \mathrm{H}_{2} \mathrm{~N}$ hydrogenbonding interactions, with the majority of the most stable complexes arising from interactions between the $\mathrm{CH}_{3}-\mathrm{C}-\mathrm{C}=\mathrm{O}$ group of MP and one of the two amine hydrogens of NEA at an
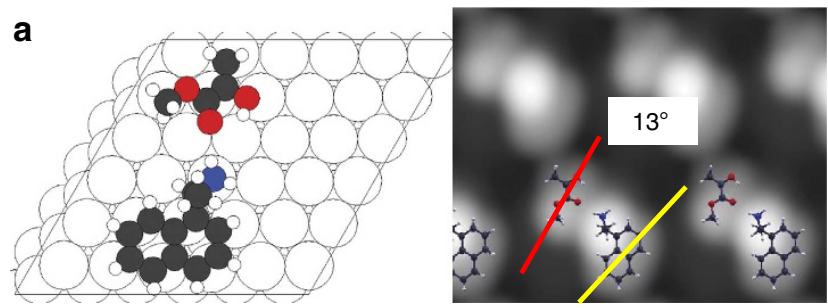

$E_{\text {int }}=-35.0 \mathrm{~kJ} \mathrm{~mol}^{-1}$, Pop $=9 \%$

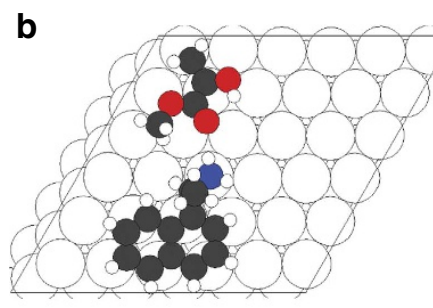

$E_{\text {int }}=-36.0 \mathrm{~kJ} \mathrm{~mol}^{-1}, \mathrm{Pop}=22 \%$

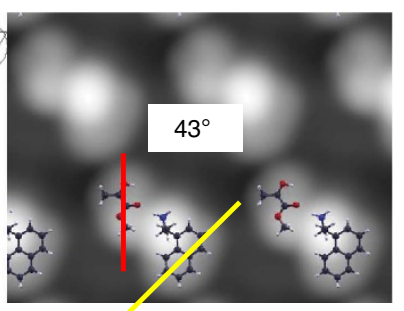

C

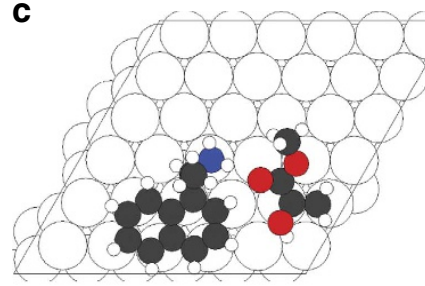

$E_{\text {int }}=-35.3 \mathrm{~kJ} \mathrm{~mol}^{-1}$, Pop $=11 \%$

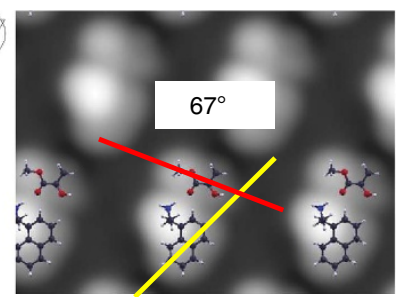

Figure 5 | Calculated structures of the docking complexes that form between exo-NEA and MP on Pd(111). Depictions of DFT-optimized

structures of the most stable docking complexes formed between MP and exo-NEA adsorbed on the dibridge[6] site, indicated as a-c. The interaction energies calculated including van der Waals' interactions are shown below each structure. The STM images simulated by the Tersoff-Hamman method are shown adjacent to each structure. The angles between the axis in the simulated images of the enol tautomer of MP, indicated by red lines, and the long axis of the naphthyl group of NEA, indicated by yellow lines, are also indicated. The relative population of each type of docking complex is calculated using a Boltzmann distribution and is reported below the respective structures.

optimal hydrogen-bonding distance of $\sim 0.22 \mathrm{~nm}$ (Supplementary Fig. 20). Thus, the most stable complexes are predominantly those which allow bridge-bonding of the vinyl group while simultaneously optimizing $\mathrm{O} \cdots \mathrm{HN}$ hydrogen bonding, although dipole-dipole interactions between NEA and MP play a role (Supplementary Discussion).

The proposed docking complexes obey the three-point bonding rule found for biological systems ${ }^{55,56}$. Here, three-point bonding occurs between the two vinyl group carbons that preferentially bind to atop sites on $\mathrm{Pd}(111)$ and by a $\mathrm{C}=\mathrm{O} \cdots \cdot \mathrm{H}_{2} \mathrm{~N}$ 


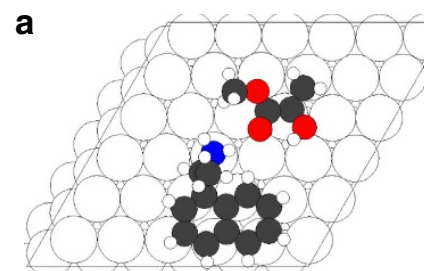

$E_{\text {int }}=-35.7 \mathrm{~kJ} \mathrm{~mol}^{-1}$, Pop $=17 \%$
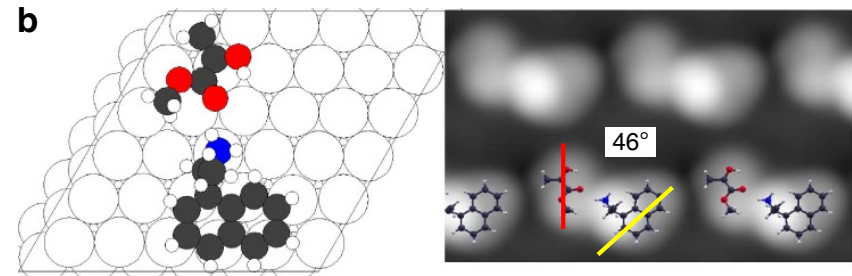

$E_{\text {int }}=-35.2 \mathrm{~kJ} \mathrm{~mol}^{-1}, \mathrm{Pop}=10 \%$

Figure 6 | Calculated structures of the docking complexes that form between endo-NEA and MP on Pd(111). Depictions of DFT-optimized structures of the most stable docking complexes formed between MP and endo-NEA adsorbed on the dibridge[6] site, indicated as $\mathbf{a}$ and $\mathbf{b}$. The interaction energies calculated including van der Waals' interactions are shown below each structure. The STM images simulated by the TersoffHamman method are shown adjacent to each structure. The angles between the axis in the simulated images of the enol tautomer of MP, indicated by red lines, and the long axis of the naphthyl group of NEA, indicated by yellow lines, are also indicated. The relative population of each type of docking complex is calculated using a Boltzmann distribution and is reported below the respective structures.

hydrogen-bonding interaction between the enol MP tautomer and an amine group on the chiral centre. Three-point bonding has also been observed between $R$-glycidol adsorbed on $S, S$-bitartratemodified $\mathrm{Pd}(111)^{57}$. Here, the epoxide oxygen on glycidol adsorbs on a palladium atop site which interacts by a simultaneous hydrogen donation from the $\mathrm{CH}_{2}-\mathrm{OH}$ group to the carboxylate, and a hydrogen acceptor interaction between the $\mathrm{CH}_{2}-\mathrm{OH}$ group and a hydroxyl group of the bitartrate. Thus, two of the three bonds in the NEA-MP interaction are to the surface and one with the modifier, while in the glycidol-bitartrate system, glycidol binds to the surface through the epoxide oxygen to an atop palladium site, with two stereo-directing interactions with the modifier. Chemisorption energies to surfaces are typically stronger than hydrogen-bonding interactions, so that surface bonding dictates the most stable locations of both the chiral modifier and chiral species or prochiral reactants. The most favourable one-to-one interactions of a chiral modifier with a chiral probe (as in the case of glycidol) or a prochiral reactant (in the case of MP) are predominantly influenced by the way in which their binding to the surface controls the stereochemistry of the weaker hydrogen-bonding interactions between them. Such a molecular-level understanding of chiral and diastereomeric interactions with chiral modifiers will lead to the rational design of heterogeneous chiral catalysts.

The interaction energies of the most stable docking complexes on $\mathrm{Pd}(111)$ vary between $\sim 35$ and $37 \mathrm{~kJ} \mathrm{~mol}^{-1}$ (Figs 5 and 6) while the fluorinated keto analogue of MP, methyl 3,3,3trifluoropyruvate (MTFP), where enol formation is suppressed ${ }^{36}$, interacts more strongly (with interaction energies between $\sim 52$ and $\sim 62 \mathrm{~kJ} \mathrm{~mol}^{-1}$ ) with NEA on $\operatorname{Pt}(111)^{45}$. This emphasizes the importance of either the substrate or fluorination in controlling complexation and, in the case of MTFP, docking complexes are observed at $\sim 300 \mathrm{~K}$ on platinum, while lower temperatures $(\sim 120 \mathrm{~K})$ are needed to observe the complexes on palladium.

The stabilization of the enol tautomer results in the formation of a $\mathrm{C}=\mathrm{C}$ double bond that hydrogenates more easily than does a carbonyl group, resulting in enhanced MP hydrogenation on NEA-modified $\operatorname{Pd}(111)$ (Fig. 1). Ethylene hydrogenates by a sequential addition of atomic hydrogen, by the so-called HoriutiPolanyi mechanism ${ }^{58}$. H/D exchange occurs through the halfhydrogenated ethyl intermediate, which repeatedly undergoes $\beta$-hydride elimination reactions to incorporate deuterium. Ethane is formed by hydrogenating the ethyl intermediate. A similar mechanism for hydrogenation of the vinyl group of the enol tautomer of MP implies that the enhancement in H/D exchange in MP can be ascribed to its stabilization by NEA, and the enhancement in methyl lactate formation indicates that the halfhydrogenated MP is also stabilized.

Stable docking complexes are found to form between the enol tautomer of MP with both the exo and endo rotamers of NEA, predicted both by first-principles DFT calculations that include van der Waals interactions and is also confirmed by comparing experimental STM images with Tersoff-Hamman simulations of the calculated structures ${ }^{52}$. The formation of the complexes is primarily dictated by the surface ensemble requirements of binding the carbon-carbon double bond to the surface while simultaneously optimizing $\mathrm{C}=\mathrm{O} \cdots \cdot \mathrm{H}_{2} \mathrm{~N}$ hydrogen-bonding interactions that predominantly stabilizes the enol tautomer in pro- $R$ configurations on an $R$-NEA-modified surface. The stabilization of the enol tautomer results in enhanced hydrogenation of MP to methyl lactate due to the presence of a more easily hydrogenated $\mathrm{C}=\mathrm{C}$ double bond. MP hydrogenation, therefore, essentially proceeds in two steps; an initial stabilization of the enol tautomer of MP to form a $\mathrm{C}-\mathrm{OH}$ single bond and a $\mathrm{C}=\mathrm{C}$ double bond, followed by facile $\mathrm{C}=\mathrm{C}$ bond hydrogenation to yield MP. The combination of chiral-NEA driven diastereomeric docking with a tautomeric preference causes an enhancement of hydrogenation activity that will lead to increased enantiomeric excesses in the catalytic reaction for chirally modified surfaces, as found experimentally. This model provides a rationale for the catalytic observations in which enhanced hydrogenation rates are found for chirally modified surfaces. However, care must be taken in directly transferring observations made for model systems in ultrahigh vacuum to supported catalysts operating in more severe solution environments, where, for example, different modifier adsorption geometries may occur ${ }^{59}$.

\section{Methods}

Sample cleaning and preparation procedure. The $\mathrm{Pd}(111)$ substrate was cleaned using a standard procedure consisting of cycles of argon ion sputtering and annealing in $3 \times 10^{-8}$ Torr of oxygen at $1,000 \mathrm{~K}$. $(R)-(+)-\alpha-(1$-naphthyl)ethylamine (Acros Organics, 99\% purity) was dosed from a home-built Knudsen source described elsewhere ${ }^{30}$. Because of the high vapour pressure of NEA, the Knudsen source was cooled to $\sim 200 \mathrm{~K}$ for at least $60 \mathrm{~min}$ before dosing. MP (Alfa Aesar, $98 \%$ purity) was purified by several freeze-pump-thaw cycles. After dosing NEA, the sample was cooled to $\sim 120 \mathrm{~K}$ and held at that temperature for all imaging. MP was dosed onto the $\mathrm{Pd}(111)$ surface through a variable leak valve while maintaining a sample temperature of $\sim 120 \mathrm{~K}$.

STM imaging procedure. STM images were acquired at a sample temperature of $\sim 120 \mathrm{~K}$ using an electrochemically etched tip made from recrystallized tungsten wire. This was conditioned by a controlled interaction with a clean $\mathrm{Au}(111)$ singlecrystal surface, likely resulting in a gold-terminated tip ${ }^{60}$. Experiments were performed using a STM (RHK UHV350 dual AFM/STM) housed in an ultrahigh vacuum chamber operating at a base pressure below $2 \times 10^{-10}$ Torr following bakeout, as described elsewhere ${ }^{41}$.

TPD experiments. TPD data were collected in another chamber operating at a base pressure of $8 \times 10^{-11}$ Torr, using a linear heating rate of $2.5 \mathrm{~K} \mathrm{~s}^{-1}$, where 
desorbing species were monitored using a Dycor quadrupole mass spectrometer placed close to and in-line-of-sight of the sample.

LEED experiments. LEED data were collected in a doubly $\mu$-metal-shielded vacuum chamber to minimize extraneous magnetic fields, and pumped by means of a liquid-nitrogen trapped diffusion pump, which operated at a base pressure of $2 \times 10^{-10}$ Torr following bakeout. I/V curves were collected with a four-grid retarding-field analyzer and the diffraction patterns were recorded as a function of beam voltage using a digital camera ${ }^{54,61-63}$.

Computational methods. DFT calculations were performed with the projector augmented wave method ${ }^{64,65}$ as implemented in the Vienna Ab-initio simulation package (VASP) (refs 66-68). The exchange-correlation potential was described using the generalized gradient approximation of Perdew, Burke and Ernzerhof ${ }^{69}$, and hydrogen-bonding interactions are reasonably well reproduced (within $\sim 4 \mathrm{~kJ} \mathrm{~mol}^{-1}$ ) using this functional, although the accuracy deteriorates as the hydrogen bonds deviate from linear ${ }^{70}$. A cutoff of $400 \mathrm{eV}$ was used for the planewave basis set, and the wavefunctions and electron density were converged to within $1 \times 10^{-5} \mathrm{eV}$. The first Brillouin zone was sampled with a $3 \times 3 \times 1$ $\Gamma$-centred k-point mesh. Geometric relaxations were considered to be converged when the force was $<0.02 \mathrm{eV} \AA^{-1}$ on all unrestricted atoms. Van der Waals corrections were included by using the method of Tkatchenko and Scheffler ${ }^{51}$ STM topography simulations were performed with the Tersoff-Hamman ${ }^{52}$ approach as implemented in bSKAN 3.7, which has been found to reproduce the shapes of experimental STM images for MP on $\operatorname{Pd}(111)^{38}$ as well as those obtained using the more rigorous Bardeen approach ${ }^{71}$.

Data availability. The data that support the findings of this study are available from the authors upon request.

\section{References}

1. Thayer, A. M. Centering on chirality. C\&E News 85, 11-19 (2007).

2. Knowles, W. S. Asymmetric hydrogenations (Nobel Lecture). Angew. Chem. Int. Ed. 41, 1998-2007 (2002).

3. Kolb, H. C. \& Sharpless, K. B. In: Transition Metals for Organic Synthesis. (eds Beller, M. \& Bolm, C.) (Wiley-VCH, 1998).

4. Sharpless, K. B. Searching for new reactivity (Nobel Lecture). Angew. Chem. Int. Ed. 41, 2024-2032 (2002).

5. Noyori, R. Asymmetric catalysis: science and opportunities (Nobel Lecture). Angew. Chem. Int. Ed. 41, 2008-2022 (2002).

6. Gaunt, M. J., Johansson, C. C. C., McNally, A. \& Vo, N. T. Enantioselective organocatalysis. Drug Discov. Today 12, 8-27 (2007).

7. McMorn, P. \& Hutchings, G. J. Heterogeneous enantioselective catalysts: strategies for the immobilisation of homogeneous catalysts. Chem. Soc. Rev. 33, 108-122 (2004)

8. Song, C. E. \& Lee, S.-G. Supported chiral catalysts on inorganic materials. Chem. Rev. 102, 3495-3524 (2002).

9. Corma, A. \& Garcia, H. Crossing the borders between homogeneous and heterogeneous catalysis: Developing recoverable and reusable catalytic systems. Top. Catal. 48, 8-31 (2008).

10. Gellman, A., Tysoe, W. \& Zaera, F. Surface chemistry for enantioselective catalysis. Catal. Lett. 145, 220-232 (2015).

11. Mahapatra, M. et al. Formation of chiral self-assembled structures of amino acids on transition-metal surfaces: alanine on $\operatorname{Pd}(111)$. J. Phys. Chem. C 118, 6856-6865 (2014)

12. Orito, Y., Imai, S. \& Niwa, S. Asymmetric hydrogenation of $\alpha$-keto esters using a platinum-alumina catalyst modified with cinchona alkaloid. J. Chem. Soc. Jpn 37, 670-672 (1980).

13. Meheux, P. A., Ibbotson, A. \& Wells, P. B. Enantioselective hydrogenation. II. Variation of activity and optical yield with experimental variables in methyl pyruvate hydrogenation catalyzed by cinchona-modified platinum/silica (EUROPT-1). J. Catal. 128, 387-396 (1991).

14. Blaser, H. U., Jalett, H. P., Muller, M. \& Studer, M. Enantioselective hydrogenation of alpha-ketoesters using cinchona modified platinum catalysts and related systems: a review. Catal. Today 37, 441-463 (1997).

15. Pfaltz, A. \& Heinz, T. Enantioselective hydrogenation of ethyl pyruvate over $\mathrm{Pt} / \mathrm{alumina}$ - systematic variation of the modifier structure. Top. Catal. 4, 229-239 (1997)

16. LeBlond, C., Wang, J., Andrews, A. T. \& Sun, Y.-K. Establishment and maintenance of an optimal chiral surface in cinchona-modified $1 \% \mathrm{Pt} / \mathrm{Al}_{2} \mathrm{O}_{3}$ for enantioselective hydrogenation of $\alpha$-keto esters.. Top. Catal. 13, 169-174 (2000).

17. Blaser, H. U., Jalett, H. P., Lottenbach, W. \& Studer, M. Heterogeneous enantioselective hydrogenation of ethyl pyruvate catalyzed by cinchonamodified Pt catalysts: effect of modifier structure. J. Am. Chem. Soc. 122, 12675-12682 (2000)
18. Kacprzak, K. \& Gawroński, J. Cinchona alkaloids and their derivatives: versatile catalysts and ligands in asymmetric synthesis. Synthesis 0961-0998 (2001).

19. Vayner, G., Houk, K. N. \& Sun, Y.-K. Origins of enantioselectivity in reductions of ketones on cinchona alkaloid modified platinum. J. Am. Chem. Soc. 126, 199-203 (2004).

20. Burgi, T. \& Baiker, A. Heterogeneous enantioselective hydrogenation over cinchona alkaloid modified platinum: mechanistic insights into a complex reaction. Acc. Chem. Res. 37, 909-917 (2004).

21. Diezi, S., Hess, M., Orglmeister, E., Mallat, T. \& Baiker, A. Chemo and enantioselective hydrogenation of fluorinated ketones on platinum modified with (R)-1-(1-naphthyl)ethylamine derivatives. J. Mol. Catal. A-Chem. 239, 49-56 (2005).

22. Blaser, H.-U. \& Studer, M. Cinchona-modified platinum catalysts: from ligand acceleration to technical processes. Acc. Chem. Res. 40, 1348-1356 (2007).

23. Mink, L. et al. The physico-chemical properties of cinchona alkaloids responsible for their unique performance in chiral catalysis. Top. Catal. 48, 120-127 (2008)

24. Talas, E., Margitfalvi, J. L. \& Egyed, O. Additional data to the origin of rate enhancement in the enantioselective hydrogenation of activated ketones over cinchonidine modified platinum catalyst. J. Catal. 266, 191-198 (2009).

25. Garland, M. \& Blaser, H. U. A heterogeneous ligand-accelerated reaction enantioselective hydrogenation of ethyl pyruvate catalyzed by cinchonamodified platinum/aluminum oxide catalysts. J. Am. Chem. Soc. 112, 7048-7050 (1990).

26. Vermeer, W. A. H., Fulford, A., Johnston, P. \& Wells, P. B. New enantioselective reactions catalysed by cinchonidine-modified platinum. J. Chem. Soc. Chem. Commun. 13, 1053-1054 (1993).

27. Mallat, T., Orglmeister, E. \& Baiker, A. Asymmetric catalysis at chiral metal surfaces. Chem. Rev. 107, 4863-4890 (2007).

28. Heinz, T. et al. 1-(1-Naphthyl)Ethylamine and derivatives thereof as chiral modifiers in the enantioselective hydrogenation of ethyl pyruvate over Ptalumina. J. Chem. Soc. Chem. Commun. 14, 1421-1422 (1995).

29. Minder, B. et al. Enantioselective hydrogenation of ethyl pyruvate over Pt/alumina modified by (R)-1-(1-naphthyl)ethylamine derivatives. J. Catal. 160, 261-268 (1996).

30. Bonello, J. M., Williams, F. J. \& Lambert, R. M. Aspects of enantioselective heterogeneous catalysis: structure and reactivity of (S)-(-)-1-(1naphthyl)ethylamine on Pt\{111\}. J. Am. Chem. Soc. 125, 2723-2729 (2003).

31. Boscoboinik, J. A., Bai, Y., Burkholder, L. \& Tysoe, W. T. Structure and distribution of S-alpha-(1-Naphthyl)-ethylamine on Pd(111). J. Phys. Chem. C 115, 16488-16494 (2011)

32. Burkholder, L., Garvey, M., Weinert, M. \& Tysoe, W. T. Structure of methyl pyruvate and alpha-(1-Naphthyl)ethylamine on $\operatorname{Pd}(111)$. J. Phys. Chem. C 115, 8790-8797 (2011).

33. Sholl, D. S. Adsorption of chiral hydrocarbons on chiral platinum surfaces. Langmuir 14, 862-867 (1998).

34. Baddeley, C. J. Fundamental investigations of enantioselective heterogeneous catalysis. Top. Catal. 25, 17-28 (2003).

35. Lavoie, S., Laliberte, M. A. \& McBreen, P. H. Adsorption states and modifiersubstrate interactions on $\mathrm{Pt}(111)$ relevant to the enantioselective hydrogenation of alkyl pyruvates in the Orito reaction. J. Am. Chem. Soc. 125, 15756-15757 (2003).

36. Lavoie, S., Laliberté, M.-A., Mahieu, G., Demers-Carpentier, V. \& McBreen, P Keto-enol driven assembly of methyl pyruvate on $\mathrm{Pt}(111)$. J. Am. Chem. Soc. 129, 11668-11669 (2007).

37. Burkholder, L. \& Tysoe, W. T. Structure and reaction pathways of methyl pyruvate on Pd(111). J. Phys. Chem. C 113, 15298-15306 (2009).

38. Garvey, M. et al. Identifying molecular species on surfaces by scanning tunneling microscopy: methyl pyruvate on $\operatorname{Pd}(111)$. J. Phys. Chem. C 117, 4505-4514 (2013).

39. Demers-Carpentier, V. et al. Direct observation of molecular preorganization for chirality transfer on a catalyst surface. Science 334, 776-780 (2011).

40. Claus, P. Selective hydrogenation of $\alpha, \beta$-unsaturated aldehydes and other $\mathrm{C}=\mathrm{O}$ and $\mathrm{C}=\mathrm{C}$ bonds containing compounds. Top. Catal. 5, 51-62 (1998).

41. Burkholder, L., Stacchiola, D., Boscoboinik, J. A. \& Tysoe, W. T. Enantioselective chemisorption on model chirally modified surfaces: 2-Butanol on alpha-(1-Naphthyl)ethylamine/Pd(111). J. Phys. Chem. C 113, 13877-13885 (2009).

42. Stacchiola, D., Azad, S., Burkholder, L. \& Tysoe, W. T. An Investigation of the reaction pathway for ethylene hydrogenation on $\mathrm{Pd}(111)$. J. Phys. Chem. B 105, 11233-11239 (2001).

43. Zaera, F. Mechanisms for ethylene hydrogenation and hydrogen-deuterium exchange over platinum(111). J. Phys. Chem. 94, 5090-5095 (1990).

44. Santarossa, G., Iannuzzi, M., Vargas, A. \& Baiker, A. Adsorption of naphthalene and quinoline on Pt, Pd and Rh: A DFT study. Chemphyschem 9, 401-413 (2008).

45. Demers-Carpentier, V. et al. Stereodirection of an $\alpha$-ketoester at sub-molecular sites on chirally modified Pt(111): Heterogeneous asymmetric catalysis. J. Am. Chem. Soc. 135, 9999-10002 (2013). 
46. Pendry, J. B. Low Energy Electron Diffraction: The Theory And Its Application To Determination Of Surface Structure (Academic Press, 1974).

47. Lavoie, S. \& McBreen, P. H. Evidence for $\mathrm{C}-\mathrm{H} \cdots \mathrm{O}=\mathrm{C}$ bonding in coadsorbed aromatic - carbonyl systems on Pt(111). J. Phys. Chem. B 109, 11986-11990 (2005)

48. Lavoie, S., Laliberte, M. A., Temprano, I. \& McBreen, P. H. A generalized twopoint $\mathrm{H}$-bonding model for catalytic stereoselective hydrogenation of activated ketones on chirally modified platinum. J. Am. Chem. Soc. 128, 7588-7593 (2006).

49. Laliberté, M.-A., Lavoie, S., Hammer, B., Mahieu, G. \& McBreen, P. H. Activation in prochiral reaction assemblies on $\mathrm{Pt}(111)$. J. Am. Chem. Soc. 130, 5386-5387 (2008).

50. Liu, W. et al. Benzene adsorbed on metals: concerted effect of covalency and van der Waals bonding. Phys. Rev. B 86, 245405 (2012).

51. Tkatchenko, A. \& Scheffler, M. Accurate molecular Van der Waals interactions from ground-state electron density and free-atom reference data. Phys. Rev. Lett. 102, 073005 (2009).

52. Tersoff, J. \& Hamann, D. R. Theory of the scanning tunneling microscope. Phys. Rev. B 31, 805-813 (1985).

53. Hall, T. J., Johnston, P., Vermeer, W. A. H., Watson, S. R. \& Wells, P. B. Enantioselective hydrogenation catalysed by palladium. Stud. Surf. Sci. Catal. 101, 221-230 (1996).

54. Zheng, T., Stacchiola, D., Poon, H. C., Saldin, D. K. \& Tysoe, W. T. Determination of the structure of disordered overlayers of ethylene on clean and hydrogen-covered $\mathrm{Pd}(111)$ by low-energy electron diffraction. Surf. Sci. 564, 71-78 (2004)

55. Easson, L. H. \& Stedman, E. Studies on the relationship between chemical constitution and physiological action: molecular dissymmetry and physiological activity. Biochem. J. 27, 1257-1266 (1933).

56. Ogston, A. G. Interpretation of experiments on metabolic processes, using isotopic tracer elements. Nature 162, 963 (1948).

57. Mahapatra, M. \& Tysoe, W. Chemisorptiveenantioselectivity of chiral epoxides on tartaric-acid modified Pd(111); three-point bonding. Phys. Chem. Chem. Phys. 17, 5450-5458 (2015).

58. Horiuti, I. \& Polanyi, M. Exchange reactions of hydrogen on metallic catalysts. Trans. Faraday Soc. 30, 1164-1172 (1934).

59. Gordon, A. D. \& Zaera, F. Adsorption of 1-(1-Naphthyl)ethylamine from solution onto platinum surfaces: implications for the chiral modification of heterogeneous catalysts. Angew. Chem. Int. Ed. 52, 3453-3456 (2013).

60. Landman, U., Luedtke, W. D., Burnham, N. A. \& Colton, R. J. Atomistic mechanisms and dynamics of adhesion, nanoindentation, and fracture. Science 248, 454-461 (1990).

61. Zheng, T., Tysoe, W. T., Poon, H. C. \& Saldin, D. K. Structure determination of ordered and disordered organic molecules on a surface from the substrate diffraction spots in low energy electron diffraction: $(\sqrt{3} \times \sqrt{3}) \mathrm{R} 30^{\circ}-\mathrm{C}_{2} \mathrm{H}_{2}$ and disordered $\mathrm{CH}_{3} \mathrm{OH}$ on $\mathrm{Pd}(111)$. Surf. Sci. 543, 19-28 (2003).

62. Poon, H. C. et al. Structure determination of disordered organic molecules on surfaces from the Bragg spots of low-energy electron diffraction and total energy calculations. Phys. Rev. B 69, 035401 (2004).

63. Zheng, T. et al. The structure of formate species on $\operatorname{Pd}\left(\begin{array}{lll}1 & 1 & 1\end{array}\right)$ calculated by density functional theory and determined using low energy electron diffraction. Surf. Sci. 574, 166-174 (2005)
64. Blöchl, P. E. Projector augmented-wave method. Phys. Rev. B 50, 17953-17979 (1994).

65. Kresse, G. \& Joubert, D. From ultrasoft pseudopotentials to the projector augmented-wave method. Phys. Rev. B 59, 1758-1775 (1999).

66. Kresse, G. \& Furthmüller, J. Efficient iterative schemes for ab initio total-energy calculations using a plane-wave basis set. Phys. Rev. B 54, 11169-11186 (1996)

67. Kresse, G. \& Furthmüller, J. Efficiency of ab-initio total energy calculations for metals and semiconductors using a plane-wave basis set. Comput. Mater. Sci. 6 , 15-50 (1996)

68. Kresse, G. \& Hafner, J. Ab initio molecular dynamics for liquid metals. Phys. Rev. B 47, 558-561 (1993).

69. Perdew, J. P., Burke, K. \& Ernzerhof, M. Generalized gradient approximation made simple. Phys. Rev. Lett. 77, 3865-3868 (1996).

70. Ireta, J., Neugebauer, J. \& Scheffler, M. On the accuracy of DFT for describing hydrogen bonds:dependence on the bond directionality. J. Phys. Chem. A 108, 5692-5698 (2004).

71. Bardeen, J. Tunnelling from a many-particle point of view. Phys. Rev. Lett. 6, 57-59 (1961)

\section{Acknowledgements}

We gratefully acknowledge support of this work by the US Department of Energy, Division of Chemical Sciences, Office of Basic Energy Sciences, under grant numbers DEFG02-03ER15474 and DE-SC008703.

\section{Author contributions}

W.T.T. conceived the project, M.M. and Y.B. collected the STM images, M.M. and M.G. carried out the DFT calculations, L.B. obtained the TPD data, M.M. measured the LEED I/V curves, D.K.S. analysed the LEED data, and W.T.T. and M.M. wrote the manuscript

\section{Additional information}

Supplementary Information accompanies this paper at http://www.nature.com/ naturecommunications

Competing financial interests: The authors declare no competing financial interests.

Reprints and permission information is available online at http://npg.nature.com/ reprintsandpermissions/

How to cite this article: Mahapatra, M. et al. Enhanced hydrogenation activity and diastereomeric interactions of methyl pyruvate co-adsorbed with $R$-1-(1-Naphthyl) ethylamine on Pd(111). Nat. Commun. 7:12380 doi: 10.1038/ncomms12380 (2016).

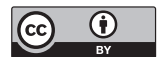

This work is licensed under a Creative Commons Attribution 4.0 International License. The images or other third party material in this article are included in the article's Creative Commons license, unless indicated otherwise in the credit line; if the material is not included under the Creative Commons license, users will need to obtain permission from the license holder to reproduce the material. To view a copy of this license, visit http://creativecommons.org/licenses/by/4.0/

C) The Author(s) 2016 